\title{
Multi-dimensional synthetic space and state measurement with spectral photonic lattices
}

\author{
Kai Wang ${ }^{1}$, James Titchener ${ }^{1}$, Bryn Bell ${ }^{2}$, Alexander S. Solntsev ${ }^{3,1}$, \\ Dragomir N. Neshev ${ }^{1}$, Benjamin J. Eggleton ${ }^{2}$, and Andrey A. Sukhorukov ${ }^{1}$ \\ ${ }^{1}$ Nonlinear Physics Centre, Research School of Physics and Engineering, \\ The Australian National University, Canberra, ACT 2601, Australia \\ ${ }^{2}$ Centre for Ultrahigh Bandwidth Devices for Optical Systems (CUDOS), Institute of Photonics and Optical Science \\ (IPOS), School of Physics, University of Sydney, Sydney, NSW 2006, Australia \\ ${ }^{3}$ School of Mathematical and Physical Sciences, University of Technology Sydney, Ultimo, NSW 2007 Australia
}

\begin{abstract}
We propose and experimentally realize spectral photonic lattices with pumpinduced frequency couplings, which can emulate multi-dimensional dynamics with synthetic gauge fields and enable single-shot measurement of the signal phase and coherence. (c) 2018 The Author(s)

OCIS codes: (190.4223) Nonlinear wave mixing; (270.5585) Quantum information and processing
\end{abstract}

The processing of information encoded in optical spectra and frequency combs [1] has multiple applications for both classical and quantum states of light ranging from communications to spectroscopy. Whereas this conventionally involves spectral demultiplexing and electro-optic conversion, there is a strong interest in fully optical approaches for ultra-fast processing in integrated platforms. Motivated by this vision, all-optical flexible spectral comb reshaping through pump-induced tunable coupling between the discrete frequency components co-propagating in a nonlinear waveguide has been recently demonstrated [2]. Here, we suggest and show experimentally, for the first time, novel applications of such spectral photonic lattices for emulating non-reciprocal multi-dimensional dynamics with synthetic gauge field and for single-shot measurement of the amplitudes, phases, and coherence between spectral lines.

First, we demonstrate that the evolution of an optical spectral comb, propagating in a single nonlinear waveguide, can emulate the wave dynamics in multi-dimensional lattices. Our work presents the first all-optical experimental realization, also providing a nontrivial generalization of previous theoretical proposals [3,4]. In our approach, a discrete signal spectrum is modulated by stronger pumps co-propagating in a nonlinear waveguide or fiber with $\operatorname{Kerr-type}\left(\chi^{(3)}\right)$ nonlinearity [see Fig. 1(a)]. The group-velocity dispersion is optimized to allow frequency conversion by four-wave mixing Bragg scattering with a large acceptance bandwidth, so that pumps induce coupling between many spectral

(a)

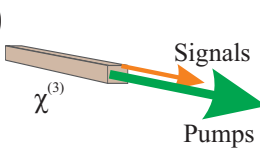

(b) Spectral lattice

(c)

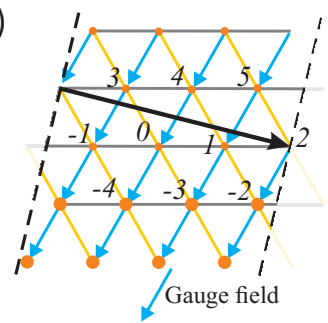

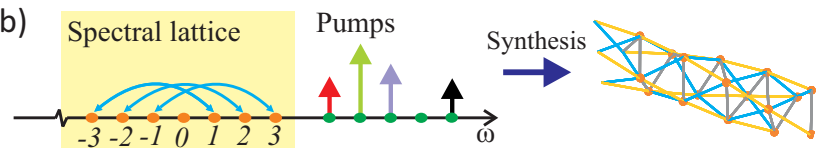

Spectral lattice Pumps

(d)
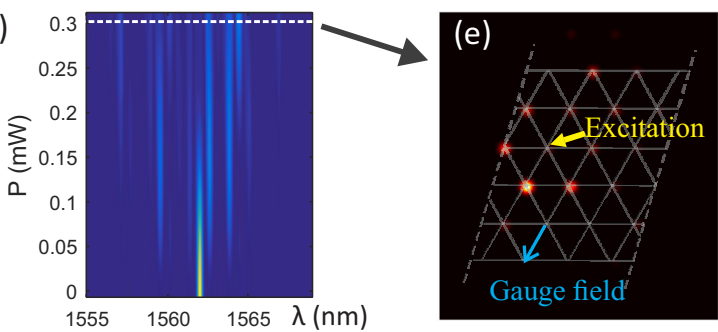

Fig. 1. (a) The concept of signal modulation by pumps in a $\chi^{(3)}$ waveguide. (b) Left: the pump and signal lattice spectra, where lines indicate the pump-induced couplings between the signals. Right: a tube of triangular lattice, which wave dynamics can be emulated in a spectral lattice. (c) Foldedout plot of a tube of triangular lattice. Black arrow show a wrapping of sites at periodic boundaries indicated with dashed black lines. Lines between neighboring lattice sites indicate couplings corresponding to the spectral lattice, blue arrows show the synthetic gauge field due to complex coupling. (d) Experimental observation of spectral lattice evolution vs. the total pump power. (e) Experimental data represented on a synthetic triangular lattice for a pump power $P=0.3 \mathrm{~mW}$. 
lines [Fig. 1(b, left)]. Remarkably, we establish that the presence of higher-order nonlocal couplings between spectral lines which are further apart can be exactly mapped to wave dynamics in complex multi-dimensional lattices with only local coupling, such as in Fig. 1(b, right).

As a representative example, we consider a lattice with $C_{1}: C_{2}: C_{3}: C_{4} \approx 2: 0:-2: i$, where $C_{n}$ is a coupling between the pairs of signal spectral lines shifted by $n$ lattice sites. This represents the wave dynamics on a tube of triangular lattice as sketched in Fig. 1(b, right), under particular numbering of sites and with periodic boundary conditions as shown in Fig. 1(c). The corresponding long-range couplings in the spectral lattice and the nearest-neighbour couplings in the synthetic triangular lattice are marked by the same colors in Figs. 1(b) and (c), respectively. Importantly, the presence of a complex-valued coupling $C_{4}$ gives rise to a synthetic gauge field in a triangular lattice, shown with blue arrows. Indeed, a circular path through three neighbouring lattice sites leads to a nontrivial phase accumulation of $\phi= \pm \pi / 2$, with the sign depending on the propagation direction. We realize the lattice experimentally in an optical fiber [2] and present in Fig. 1(d) the output spectrum versus pump power, with the latter emulating the time dynamics. We map the experimental data to the corresponding synthetic lattice in Fig. 1(e), and observe a directional transport away from the input site attributed to the presence of a gauge field and associated time-reversal symmetry breaking. Importantly, this approach is scalable to higher-dimensional synthetic lattices by designing the nonlocal couplings.
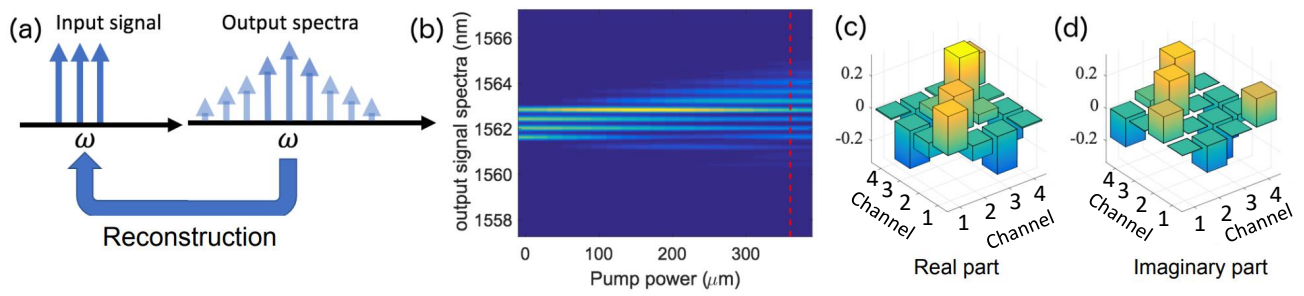

Fig. 2. (a) Single-shot measurement of the output spectral intensity, after a signal evolution with optimized pump-induced couplings, enables full reconstruction of the input signal, including spectral amplitudes, phases, and coherence. (b) Experimentally measured output spectra vs. the pump power for the input state $\left|\omega_{1}\right\rangle+i\left|\omega_{2}\right\rangle-\left|\omega_{3}\right\rangle-i\left|\omega_{4}\right\rangle$. (c) Real and (d) imaginary parts of the input density matrix, reconstructed from the output spectra for $360 \mu \mathrm{W}$ pump power [red dotted line in (b)], with the fidelity of $93.9 \%$.

In the second application, we suggest and demonstrate experimentally that a lattice with pump-induced nonlocal spectral couplings can enable complete reconstruction of the input spectra, including information on the phase and coherence, by a single-shot spectral intensity measurement at the output [Fig. 2(a)]. The input state is spread out in the spectral lattice to more sites than the unknowns in its density matrix, and we specially design the couplings to facilitate robust reconstruction with the lowest sensitivity to measurement errors. In experiment, we demonstrate the reconstruction of input states composed of four frequency channels. The measured evolution and broadening of the spectrum is shown in Fig. 2(b). We then use the output spectrum at an optimal power level to reconstruct the complex density matrix [Figs. 2(c,d)], which then fully defines the complex amplitudes and coherence of all the input spectral lines. This approach is scalable and can be extended to a larger number of spectral lines.

To summarize, we outline novel applications of spectral photonic lattices, which can be simply realized in a nonlinear waveguide through all-optical pump modulation of signal spectra. We show that pump-induced local and nonlocal couplings enable emulation of multi-dimensional dynamics on lattices of complex geometries with synthetic gauge field, and can also find applications for single-shot reconstruction of the complex signal spectrum and its coherence based on simple spectral intensity measurements. Furthermore, the coherent and conservative nature of the implemented lattice opens prospects for quantum applications including simulations, information processing, and tomographic reconstruction through flexible manipulation of single and multi-photon states with spectral encoding.

This work was supported by the Australian Research Council: Discovery Project (DP160100619); Centre of Excellence CUDOS (CE110001018); Laureate Fellowship (FL120100029).

\section{References}

1. T. J. Kippenberg, R. Holzwarth, et al., Science 332, 555 (2011).

2. B. A. Bell, K. Wang, et al., Optica 4, 1433 (2017).

3. J. Casanova, A. Mezzacapo, et al., Phys. Rev. Lett. 108, 190502 (2012).

4. L. Yuan, M. Xiao, et al., arXiv 1710.01373 (2017). 